\title{
PROPAGATION OF A SHOCK WAVE IN A RADIATING SPHERICALLY SYMMETRIC DISTRIBUTION OF MATTER
}

\author{
L. HERRERA \\ Departamento de Fisica, Facultad de Ciencias, Universidad Central de Venezuela \\ AND \\ L. NÚÑEZ \\ Departamento de Fisica, Facultad de Ciencias, Universidad Central de Venezuela, and \\ Laboratorio de Fisica Teórica, Departamento de Fisica, Facultad de Ciencias, Universidad de Los Andes \\ Received 1986 October 2; accepted 1987 January 30
}

\begin{abstract}
A method used to study the evolution of radiating spheres reported by L. Herrera, J. Jiménez, and G. Ruggeri in 1980 is extended to the case in which the sphere is divided in two regions by a shock wave front. The equations of state at both sides of the shock are different, and the solutions are matched on it via the Rankine-Hugoniot conditions. The outer region metric is matched with a Vaidya solution on the boundary surface of the sphere. As an example of the procedure, we consider two known solutions for radiating systems. The matter distribution is free of singularities everywhere within the sphere and a Gaussian-like pulse is assumed to carry out a fraction of the total mass. Exploding models are then obtained. Finally, the results are discussed in the light of recent work on gravitational collapse and supernovae.

Subject headings: shock waves - stars: supernovae
\end{abstract}

\section{INTRODUCTION}

The problem of gravitational collapse has been the subject of lengthy discussions. A large number of different techniques and approaches have been introduced both in Newtonian and in general relativistic calculations. A special interest arises in relation with questions on the outcome of the gravitational collapse and, more specifically with the possibility of modeling a Type II supernovae (Arnett 1966, 1979; Bethe and Wilson; Brown, Bethe, and Baym 1982; Colgate and White 1964; Schramm and Arnett 1975; Schwartz 1967; Van Riper 1979; Wilson 1971, 1979; and references therein). Despite the diversity of points of view on this problem, there are two aspects concerning the process of collapse where a concensus has emerged among almost all the researchers in the field, namely:

1. If we expect that the collapse is to lead to a compact object (neutron star or black hole), then neutrinos must be copiously emitted. This fact is independent of their possible role in the ejection of the outer mantle of the star in a supernova outburst (Kazanas and Schramm 1979).

2. A shock is formed in the process of collapse. It may appear as a result of the infall of the outer core on the more compact (halted or rebounding) inner core (Colgate and Johnson 1960; Cooperstein, Bethe, and Brown 1984; Van Riper 1980). Also if a phase transition occurs during the process of collapse (e.g., pion condensation), then the interface between the two phases may be treated as a shock (Migdal, Chernoutsan, and Mishustin 1979).

In this paper we propose a method to study a general relativistic collapse, including the two effects mentioned above (neutrinos and shock). It represents an extension of an earlier work on the same problem (Herrera, Jiménez, and Ruggeri 1980, hereafter HJR). In the present approach we consider that the sphere is divided in two regions by a shock wave front whose origin could be either hydrodynamic or a phase transition, or both. At each side of the shock there is a different equation of state, which physically is reasonable (Lichtestadt, Sack, and Bludman 1980; Van Riper 1980).

The solutions corresponding to either side of the front are matched across it via the Rankine-Hugoniot conditions (Taub 1948, 1983). The solution for the outer region is matched with the Vaidya metric on the boundary of the sphere.

Following HJR's algorithm, we introduce an heuristic assumption relating the pressure, the energy density, and the radial velocity of matter. This Ansatz, together with the junction conditions at the shock and at the boundary of the matter distribution, leads to a system of ordinary differential equations for quantities evaluated at either the boundary surface or the shock front. Thus, the numerical integration of this system allow us, using the field equations, to find the profile of the physical variables for any piece of material.

As an example of the procedure we consider two solutions which are known from HJR. The solution describing the inner part of the sphere is a generalization of the well-known Schwarzschild interior metric to the radiating, nonstatic case. For the outer region we have chosen a nonstatic generalization of the Tolman VI solution (Tolman 1939). Although this selection is completely arbitrary and was made just to illustrate the method, the two solutions above present interesting features, namely:

1. The equation of state in the inner region is stiffer than the equation of state of the outer one. Moreover, in the static limit the inner equation of state corresponds to the homogeneous Schwarzschild incompressible fluid.

2. We recall that the equation of state in the static Tolman VI model approaches that for highly compressed Fermi gas.

Thus, in spite of the arbitrariness in the choice of the equation of state at either side, we expect that some of the conclusions 
emerging from our example could still be useful and not very different from those coming out from models with more realistic equations of state.

The paper is organized as follows. A brief resumé of the HJR method, as well as the conventions used, are included in $\S$ II. In $\S$ III the Rankine-Hugoniot conditions are discussed and explicitly written in terms of the variables of our approach. At the end of $\S$ III the outline of the method is presented. A particular example is worked out in $\S$ IV to illustrate the procedure. In $\S \mathrm{V}$ the results are discussed. Finally some details of intermediate calculations are sketched in the Appendix.

\section{THE HJR METHOD}

For sake of completeness we include here a brief resumé of the HJR method, which is our starting point. Let us consider a nonstatic distribution of matter, which is spherically symmetric. In radiation coordinates (Bondi 1964) the
metric takes the form

$$
d s^{2}=e^{2 \beta}\left[(V / r) d u^{2}+2 d u d r\right]-r^{2}\left(d \theta^{2}+\sin ^{2} \theta d \varphi^{2}\right),
$$

where $\beta$ and $V$ are functions $u$ and $r$. Here $u \equiv x^{0}$ is a timelike coordinate, $r \equiv x^{1}$ is a null coordinate, and $\theta \equiv x^{2}$ and $\varphi \equiv x^{3}$ are the usual angle coordinates. In these coordinates the components of the energy momentum tensor are distinguished by a bar, while differentiation with respect to $u$ and $r$ are denoted by subscripts 0 and 1 , respectively.

Thus, it can be shown (Bondi 1964; HJR) that the Einstein equations may be written as

$$
\begin{gathered}
\frac{\rho+P \omega^{2}}{1-\omega^{2}}+\epsilon=\frac{r}{V} e^{-2 \beta} \bar{T}_{00}=\frac{1}{4 \pi r(r-2 \tilde{m})}\left(-\tilde{m}_{0} e^{-2 \beta}+\frac{r-2 \tilde{m}}{r} \tilde{m}_{1}\right), \\
\frac{\rho-P \omega}{1+\omega}=e^{-2 \beta} \bar{T}_{01}=\frac{\tilde{m}_{1}}{4 \pi r^{2}}, \\
\frac{1-\omega}{1+\omega}(\rho+P)=\frac{V}{r} e^{-2 \beta} \bar{T}_{11}=\frac{r-2 \tilde{m}}{2 \pi r^{2}} \beta_{1}, \\
P=-\bar{T}_{2}^{2}=\frac{-\beta_{01} e^{-2 \beta}}{4 \pi}+\frac{1}{8 \pi}\left(1-\frac{2 \tilde{m}}{r}\right)\left(2 \beta_{11}+4 \beta_{1}{ }^{2}-\frac{\beta_{1}}{r}\right)+\frac{3 \beta_{1}\left(1-2 \tilde{m}_{1}\right)-\tilde{m}_{11}}{8 \pi r},
\end{gathered}
$$

where $\rho, P$, and $\omega$ are the energy density, pressure, and radial velocity of matter, respectively, as measured by a local Minkowski observer. Also, the flux of radiation $\hat{\epsilon}$, as measured by the same observer, is related to $\epsilon$, by

$$
\epsilon=\hat{\epsilon} \frac{1+\omega}{1-\omega} \text {. }
$$

The field equations may be integrated outside matter to obtain

$$
\beta=0, \quad V=r-2 \tilde{m}(u), \quad \epsilon=-\frac{\tilde{m}_{0}}{4 \pi r(r-2 \tilde{m})},
$$

where $\tilde{m}$ is a function of integration depending on $u$. Inside matter the function $\tilde{m}(u)$ is generalized to $\tilde{m}(u, r)$ by setting

$$
V=e^{2 \beta}(r-2 \tilde{m}(u, r)) \text {. }
$$

everywhere.

Also note that the velocity of matter in the radiative coordinates is given by

$$
\frac{d r}{d u}=\frac{V}{r} \frac{\omega}{1-\omega}
$$

Next let us define the two auxiliary functions

$$
\begin{aligned}
\tilde{\rho} & \equiv \frac{\rho-\omega P}{1+\omega}, \\
\tilde{P} & \equiv \frac{P-\omega \rho}{1+\omega},
\end{aligned}
$$

hereafter referred to as effective density and effective pressure, respectively. Observe that from fields equations (3) and (4) we have

$$
\begin{gathered}
\tilde{m}=\int_{0}^{r} 4 \pi \tau^{2} \tilde{\rho} d \tau, \\
\beta=\int_{a}^{r} \frac{2 \pi \tau^{2}}{\tau-2 \tilde{m}}(\tilde{\rho}+\tilde{P}) d \tau,
\end{gathered}
$$

Where $r=a(u)$ defines the boundary of the fluid sphere. 
Now the algorithm to construct the solution is given as follows (for details see Bondi 1964 and HJR):

1. Take a static interior solution of the Einstein equations with spherical symmetry, and given

$$
\rho_{\mathrm{st}}=\rho(r), \quad P_{\mathrm{st}}=P(r) .
$$

2. Assume that the $r$ dependence of $\tilde{P}$ and $\tilde{\rho}$ is the same as that of $P_{\text {st }}$ and $\rho_{\text {st }}$, but with the boundary condition which now, because of equation (10), reads

$$
\tilde{P}_{a}=-\omega_{a} \tilde{\rho}_{a}
$$

From now on the subscript $a$ indicates that the quantity is evaluated at the surface.

3. Using equations (11) and (12), with the $r$ dependence of $\tilde{\rho}$ and $\tilde{P}$, one gets $\tilde{m}$ and $\beta$ up to three functions of $u$, which will be specified below.

4. For these three functions one has two differential equations, one of which (eq. [8]) is evaluated at $r=a$ and the other is

$$
\left(T^{\mu}{ }_{1 ; \mu}\right)_{a}=0
$$

or which is equivalent, $P_{a}=0$.

Another $u$-dependent equation can be obtained evaluating equation (6) at $r=a+0$. Obtaining

$$
E(u)=\left(4 \pi r^{2} \epsilon\right)_{r=a+0}=\left[\frac{\tilde{m}_{0}}{(1-2 \tilde{m} / r)}\right]_{r=a+0} .
$$

Thus, one has three differential equations for four unknown functions of $u$, which are the radius $a$, the velocity of the surface, the "luminosity" $E$, and the function $\tilde{m}$ evaluated at the surface (see eqs. [15], [17], and [21]).

5. Given one of the functions, the system may be integrated for any particular initial data.

6 . Feeding back the result of integration in the expressions for $\beta$ and $\tilde{m}$, these two functions are completely determined.

7. Using equations (2) $-(5), \rho, P, \omega$, and $\epsilon$ may be found.

In order that the method outlined above should be completely consistent, it is necessary to match the interior solution with Vaidya metric at the boundary of the fluid distribution (Darmois or Lichnerowicz conditions). It is easy to check that these conditions are equivalent to the continuity of the functions $\beta$ and $\tilde{m}$ across the boundary (Herrera and Jiménez 1983) and to equation

$$
-\beta_{0 a}+\left[1-\frac{2 \tilde{m}_{a}}{a(u)}\right] \beta_{1 a}-\frac{\tilde{m}_{1 a}}{2 a(u)}=0,
$$

where, as before, we have defined the boundary by the equation $r=a(u)$.

Thus, we shall demand the continuity of $\beta$ and $\tilde{m}$ across the boundary (see eqs. [11], [12]). On the other hand, condition (13a) is completely equivalent to one of the equations at the surface (see eq. [13b] below).

Finally, the vanishing of the pressure at the boundary (which is explicitly assumed in this work) may be shown to be direct consequence of equations (13a), (8), (4), and the continuity of $\beta$ across the boundary (eq. [12]).

As it should be clear from the previous comments, the crucial point in the algorithm is the system of equations for quantities evaluated at the surface (surface equations). Two of them are the same for any model with spherically symmetric matter distribution. Thus, from equations (6) and (8) we get

$$
\dot{a}=\left(1-\frac{2 m}{a}\right) \frac{\omega_{a}}{1-\omega_{a}},
$$

with $\dot{a} \equiv d a / d u$, and where $m \equiv \tilde{m}_{a}$ is the total mass.

Scaling the radius $a$, the total mass $m$, and the timelike coordinate $u$ by the initial mass $\tilde{m}(u=0) \equiv m(0)$, we get

$$
A \equiv a / m(0), \quad \bar{M} \equiv m / m(0), \quad u / m(0) \rightarrow u,
$$

and defining

$$
F \equiv 1-2 \bar{M} / A, \quad \Omega \equiv 1 /\left(1-\omega_{a}\right)
$$

we can write equation $(13 b)$ as

$$
\dot{A}=F(\Omega-1),
$$

which is the first surface equation and, as we have mentioned before, is implied by the junction conditions.

The second surface equation relates the total mass-loss with the energy flux through the surface. This can be obtained by evaluating equation (6) for $r=a+0$ and takes the form

$$
\dot{\bar{M}}=-F E
$$

Or, using equations (14) and (15)

$$
\frac{\dot{F}}{F}=\frac{2 E+(1-F)(\Omega-1)}{A}
$$


The third equation at the surface will be obtained from the conservation equation $T_{1 ; \mu}^{\mu}=0$ evaluated at the surface. Thus, from

$$
\left(T^{\mu}{ }_{1 ; \mu}\right)_{a}=0
$$

we get

$$
-\left[\frac{(\tilde{\rho}+\tilde{P})}{(1-2 \tilde{m} / r)}\right]_{0 a}+\left(\frac{\partial \tilde{P}}{\partial r}\right)_{a}+\left[\frac{(\tilde{\rho}+\tilde{P})}{(1-2 \tilde{m} / r)}\left(4 \pi r \tilde{P}+\frac{\tilde{m}}{r^{2}}\right)\right]_{a}=\left[\frac{2}{r}(P-\tilde{P})\right]_{a},
$$

or, after a lengthy and tedious calculation,

where

$$
-\frac{\dot{\tilde{\rho}}_{a}}{\tilde{\rho}_{a}}+\frac{\dot{F}}{F}+\frac{\dot{\Omega}}{\Omega}+(\Omega-1)\left[4 \pi a \tilde{\rho}_{a} \frac{(3 \Omega-1)}{\Omega}+\Omega F \frac{\tilde{\rho}_{1 a}}{\tilde{\rho}_{a}}-\frac{(3+F)}{2 a}\right]+\frac{\Omega^{2} F R_{a}}{\tilde{\rho}_{a}}=0,
$$

$$
R_{a}(u)=\left[\frac{(\tilde{\rho}+\tilde{P})}{(1-2 \tilde{m} / r)}\left(4 \pi r \tilde{P}+\frac{\tilde{m}}{r^{2}}\right)+\frac{\partial \tilde{P}}{\partial r}\right]_{a}
$$

If the effective density $\tilde{\rho}$ is separable, i.e.

$$
\tilde{\rho}=f(u) h(r),
$$

then equation (19) becomes

$$
\frac{\dot{F}}{F}+\frac{\dot{\Omega}}{\Omega}(1-F)=Q(F, \Omega, A)
$$

with

$$
Q(F, \Omega, A)=(F-1)(\Omega-1)\left[4 \pi a \tilde{\rho}_{a} \frac{(3 \Omega-1)}{\Omega}+\frac{\Omega F \tilde{\rho}_{1 a}}{\tilde{\rho}}-\frac{(3+F)}{2 a}-K(a) F\right]+\frac{(1-F) \Omega^{2} F R_{a}(u)}{\tilde{\rho}_{a}}
$$

where

$$
K(a) \equiv \frac{d}{d a} \ln \left[\frac{1}{a} \int_{0}^{a} r^{2} \frac{h(r)}{h(a)} d r\right] .
$$

In the next section we shall consider a composite sphere, formed by two regions (each one described by a solution of the kind we have just discussed) separated by a shock wave.

III. A COMPOSITE SPHERE: MATCHING ACROSS THE SHOCK

Let us now consider a sphere of radius $a(u)$ divided in two regions (say, I and II) by a discontinuity surface (a shock) of radius $c(u)$ (see Fig. 1). Both $a(u)$ and $c(u)$ are functions of the timelike coordinate.

The matching conditions across the shock (the Rankine-Hugoniot conditions) require the continuity of the first and the second fundamental form, plus the continuity of $T_{\mu \nu} n^{v}$, where $T_{\mu \nu}$ is the energy momentum tensor and $n^{v}$ is the unit vector normal to the

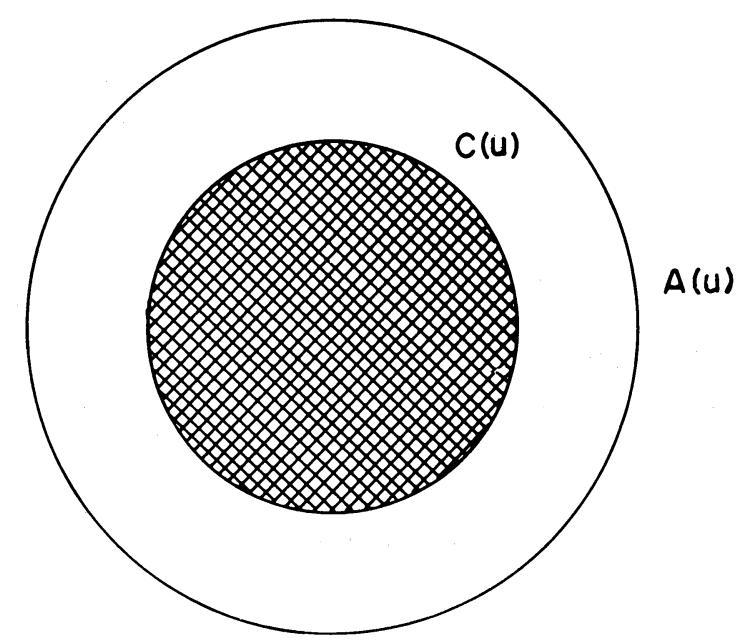

FIG. 1.-The model sphere in two regions considered through this work. The inner core, region I, is enclosed by a shock surface, $C(u)$. Region II, the outer mantle, completes the sphere of radius $A(u)$. 
surface $r=c(u)$ (Taub 1984). The continuity of the first and the second fundamental form lead to the following conditions (see Herrera and Jiménez 1983)

$$
[\beta]_{c}=[\tilde{m}]_{c}=0,
$$

$$
\left[2 \beta_{1} e^{2 \beta}(1-2 \tilde{m} / r)-2 \beta_{0}-e^{2 \beta} \tilde{m}_{1} / r\right]_{c}=0
$$

where

$$
\left.[f]_{c} \equiv f\right|_{r=c+0}-\left.f\right|_{r=c-0}
$$

Next, since $\beta$ is continuous across $c(u)$,

$$
\beta(r, u) \approx \beta(c, u)+\left.\beta_{1}\right|_{c}(r-c)
$$

and then

$$
\left[\beta_{0}+c_{0} \beta_{1}\right]_{c}=0
$$

Substituting equation (25) into equation (24), we get

$$
\left[\frac{1-\omega}{1+\omega}(\rho+P) \Psi-\frac{\rho-\omega P}{1+\omega}\right]_{c}=0,
$$

with

$$
\Psi \equiv 1+c_{0} e^{-2 \beta}(1-2 \tilde{m} / r)^{-1}
$$

Finally, the conditions

$$
\left[T_{\mu v} n^{v}\right]_{c}=0
$$

read

$$
\left[\frac{1-\omega}{1+\omega}(\rho+P) \Psi-\frac{\rho-\omega P}{1+\omega}\right]_{c}=0,
$$

which is exactly the condition (26) above, and

$$
\left[\frac{\rho+\omega^{2} P}{1-\omega^{2}}+\epsilon-\frac{\rho-\omega P}{1+\omega} \Psi\right]_{c}=0,
$$

where we have used the fact that the general form of the energy momentum tensor is given by

$$
T_{\mu v}=(\rho+P) U_{\mu} U_{v}-P g_{\mu v}+\epsilon K_{\mu} K_{v},
$$

with

$$
\begin{gathered}
U_{\mu}=e^{2 \beta} \frac{(1-2 \tilde{m} / r)^{1 / 2}}{\left(1-\omega^{2}\right)^{1 / 2}} \delta^{0}{ }_{\mu}+\left(\frac{1-\omega}{1+\omega}\right)^{1 / 2}\left(1-\frac{2 \tilde{m}}{r}\right)^{-1 / 2} \delta^{1}{ }_{\mu}, \\
K_{\mu}=e^{2 \beta}(1-2 \tilde{m} / r)^{1 / 2} \delta^{0}{ }_{\mu} .
\end{gathered}
$$

At this point the following remark is in order: since $r=c(u)$ is a surface of discontinuity, then $c_{0} \neq(d r / d u)_{c}$. In other words, the velocity of the shock, in general, is not equal to the velocity of matter evaluated at $r=c(u)$ (which is fairly obvious). Should $r=c(u)$ not be a discontinuity surface, then $c_{0}=(d r / d u)_{c}$, and it is easily found from equations (26) and $(28)$ that $[\epsilon]_{c}=[P]_{c}=0$. Actually this is the situation at the boundary surface $r=a(u)$, which obviously is not a shock. Of course, for a surface to be a boundary [e.g., $r=a(u)]$, the velocity of matter at that surface must be equal to the velocity of the surface itself.

Let us now rewrite conditions (27) and (28) in terms of the effective variables. Using equations (9) and (10), we obtain

$$
\begin{gathered}
(1-\Psi)[\tilde{\rho}]_{c}=\Psi[\tilde{P}]_{c}, \\
{\left[\Omega_{s}\left(\Omega_{s}-1\right)(\tilde{\rho}+\tilde{P})+(1-\Psi) \tilde{\rho}+\epsilon\right]_{c}=0,}
\end{gathered}
$$

with

$$
\Omega_{s}=\frac{1}{1-\omega_{c \pm 0}}
$$


Also, for the metric functions $\beta$ and $\tilde{m}$ we obtain, using the field equations (3) and (4),

$$
\begin{aligned}
\tilde{m}_{\mathrm{I}} & =\int_{0}^{r} 4 \pi \tau^{2} \tilde{\rho}_{\mathrm{I}} d \tau, \quad 0 \leq r \leq c(u) \\
\tilde{m}_{\mathrm{II}} & =\int_{c(u)}^{r} 4 \pi \tau^{2} \tilde{\rho}_{\mathrm{II}} d \tau+\tilde{m}[u, c(u)], \quad c(u) \leq r \leq a(u) ;
\end{aligned}
$$

and

$$
\begin{aligned}
& \beta_{\mathrm{I}}=\int_{c(u)}^{r} \frac{2 \pi \tau^{2}}{\tau-2 \tilde{m}_{\mathrm{I}}}\left(\tilde{\rho}_{\mathrm{I}}+\tilde{P}_{\mathrm{I}}\right) d \tau+\beta_{\mathrm{II}}[u, c(u)] ; \quad 0 \leq r \leq c(u) ; \\
& \beta_{\mathrm{II}}=\int_{a(u)}^{r} \frac{2 \pi \tau^{2}}{\tau-2 \tilde{m}_{\mathrm{II}}}\left(\tilde{\rho}_{\mathrm{II}}+\tilde{P}_{\mathrm{II}}\right) d \tau ; \quad c(u) \leq r \leq a(u) ;
\end{aligned}
$$

where the subscripts I and II indicate the region the quantity is referred to. We can now restate the HJR algorithm for the composite sphere as follows:

1. Take two static interior solutions of the Einstein equations for perfect fluids with spherical symmetry, given

$$
\begin{array}{ll}
\rho_{\text {st } 1}=\rho_{\mathrm{I}}(r) ; & \rho_{\mathrm{st} \mathrm{II}}=\rho_{\mathrm{II}}(r) ; \\
P_{\text {st I }}=P_{\mathrm{I}}(r) ; & P_{\mathrm{st} \mathrm{II}}=P_{\mathrm{II}}(r) .
\end{array}
$$

2. Assume that the $r$ dependence of $\tilde{P}_{\mathrm{I}, \mathrm{II}}$ and $\tilde{\rho}_{\mathrm{I}, \mathrm{II}}$ is the same as that of $P_{\mathrm{st}, \mathrm{II}}$ and $\rho_{\mathrm{st} \text { I,II }}$, but taking care of the boundary condition which, for this case, reads

$$
\tilde{P}_{\mathrm{II} a}=-\omega_{a} \tilde{\rho}_{\mathrm{II} a},
$$

and the conditions at $r=c(u)$ given by equations (31) and (32).

3. With the $r$ dependence of $\tilde{\rho}_{\mathrm{I}, \mathrm{II}}$ and $\widetilde{P}_{\mathrm{I}, \mathrm{II}}$, and using equations (33)-(36), one gets $\tilde{m}_{\mathrm{I}, \mathrm{II}}$ and $\beta_{\mathrm{I}, \mathrm{II}}$ up to some functions of $u$ which will be specified below.

4. For these functions of $u$ one obtains the following ordinary differential equations (surface equations):

a) The equations (15), (17), (21);

b) The matching conditions across $r=c(u)$.

5. Given one function evaluated at $r=a(u)$ and two functions evaluated at $r=c(u)$, the complete system (4a) and (4b) may be integrated for any particular data.

6. Substituting the result of integration in the expressions for $\tilde{m}_{\mathbf{I}, \mathrm{II}}$ and $\beta_{\mathbf{I}, \mathrm{II}}$, these four functions are completely determined.

7. Using equations (2)-(5), the matter variables $\rho, P, \omega$, and $\epsilon$ may be found for any part of the sphere.

In the above we have outlined the general program for the attainment of models. However, before discussing a specific example we would like to make the following observations:

1. Because of the large number of functions appearing in the system (4a) and (4b), there are many ways to achieve step (5). In the next section we are going to outline only one of them. As will be seen below, the choice depends strongly on what one considers are the observable functions of the process.

2. The fact that two functions evaluated at $r=c(u)$ should be given by hand [in contrast with the situation at $r=a(u)$, where only one function of $u$ has to be given] is related to the continuity of the energy flux across the boundary surface $r=a(u)$. Indeed, evaluating the equation (2) at both sides of $r=a(u)$ and using equations (3) and (13a), it is easily shown that the energy flux is continuous across $r=a(u)$; alternatively, one may use equations (26)-(28) at $r=a(u)$. This, of course, is not the case, in general, for the surface $r=c(u)$, as it follows from equations (27) and (28).

\section{AN EXAMPLE}

In this section we shall explicitly work out an example to illustrate the method just presented above. Let us first specify the solutions in both regions of the sphere.

\section{a) The Inner Region}

For the inner region $[0 \leq r \leq c(u)-0]$, we shall consider a solution inspired on the well-known Schwarzschild interior solution (see HJR). With this in mind, we take

$$
\tilde{\rho}_{\mathbf{I}}=f(u)
$$

and

$$
\tilde{P}_{\mathrm{I}}=f(u) \frac{g(u)\left[1-8 \pi r^{2} f(u) / 3\right]^{1 / 2}-1 / 3}{1-g(u)\left[1-8 \pi r^{2} f(u) / 3\right]^{1 / 2}}
$$

where $f(u)$ and $g(u)$ are two arbitrary functions of $u$. Observe that, unlike the one region case (HJR), the effective pressure involves in this case an additional function: $g(u)$. 
We can now integrate equations (33) and (35) to obtain

$$
\tilde{m}_{\mathrm{I}}(u, r)=\frac{4 \pi}{3} f(u) r^{3}
$$

and

$$
\beta_{\mathrm{I}}=-\frac{1}{2} \ln \left|\frac{\left[1-8 \pi r^{2} f(u) / 3\right]^{1 / 2}\left\{g(u)\left[1-8 \pi c(u)^{2} f(u) / 3\right]^{1 / 2}-1\right\}}{\left[1-8 \pi c(u)^{2} f(u) / 3\right]^{1 / 2}\left\{g(u)\left[1-8 \pi r^{2} f(u) / 3\right]^{1 / 2}-1\right\}}\right|+\beta_{\mathrm{II}}[u, c(u)] .
$$

So far we have three unknown functions of $u$ which are $f(u), g(u)$, and $\beta_{\mathrm{II}}[u, c(u)]$.

\section{b) The Outer Region}

For the outer region, also, we have chosen a solution investigated in HJR. It is a solution inspired in the static Tolman VI metric (Tolman 1939). In this case the effective variables are

$$
\begin{gathered}
\tilde{\rho}_{\mathrm{II}}=\frac{3 h(u)}{r^{2}} \\
\tilde{P}_{\mathrm{II}}=\frac{h(u)}{r^{2}}\left[\frac{1-9 k(u) r}{1-k(u) r}\right],
\end{gathered}
$$

where $h(u)$ and $k(u)$ are unknown functions of $u$, related through the boundary condition $\tilde{P}_{\mathrm{II} a}=-\omega_{a} \tilde{\rho}_{\mathrm{II}}$. Thus, we get for the metric functions

$$
\begin{gathered}
\tilde{m}_{\mathrm{II}}(u, r)=12 \pi h(u) r-4 \pi c(u)\left[3 h(u)-c(u)^{2} f(u) / 3\right], \\
\beta_{\mathrm{II}}(u, r)=\frac{8 \pi h(u)}{\gamma k(u)+\delta}\left\{2 \ln \left|\frac{1-k(u) r}{1-k(u) a(u)}\right|+\left[1+\frac{3 k(u) \gamma}{\delta}\right] \ln \left|\frac{\gamma+\delta r}{\gamma+\delta a(u)}\right|\right\},
\end{gathered}
$$

with

$$
\gamma=2\left[12 \pi c(u) h(u)-4 \pi c(u)^{3} f(u) / 3\right], \quad \delta=1-24 \pi h(u) .
$$

Having specified the solutions at either side of the shock (up to some functions of $u$ ), we are now ready to write out the surface equations. Three of them correspond to the surface at $r=a(u)$ (eqs. [15], [17], and [21]). Observe that these three equations are coupled with the solutions at $r=c(u)$ through the appearance of the function $f(u)$ in equations (43) and (44).

Next we have the Rankine-Hugoniot conditions (eqs. [31], [32]), which, taking into account equations (37), (38), and (41), (42), read

$$
1-\frac{2 \Psi}{3\left\{1-g(u)\left[1-8 \pi c(u)^{2} f(u) / 3\right]^{1 / 2}\right\}}=\frac{h(u)}{f(u) c(u)^{2}}\left\{3-\frac{4 \Psi[1-3 k(u) c(u)]}{[1-k(u) c(u)]}\right\}
$$

and

$$
\frac{2 f(u) \Omega_{\mathrm{I}}\left(\Omega_{\mathrm{I}}-1\right)}{3\left\{1-g(u)\left[1-8 \pi c(u)^{2} f(u) / 3\right]^{1 / 2}\right\}}+(1-\Psi) f(u)+\epsilon_{\mathrm{I}}=\frac{h(u)}{c(u)^{2}} \frac{4[1-3 k(u) c(u)]}{[1-k(u) c(u)]}\left(\Omega_{\mathrm{II}}-1\right) \Omega_{\mathrm{II}}+(1-\Psi) \frac{3 h(u)}{c(u)^{2}}+\epsilon_{\mathrm{II}}
$$

where

$$
\Omega_{\mathrm{I}} \equiv \frac{1}{1-\omega_{\mathrm{I} c}} \equiv\left(\frac{1}{1-\omega_{\mathrm{I}}}\right)_{r=c-0}, \quad \Omega_{\mathrm{II}} \equiv \frac{1}{1-\omega_{\mathrm{II} c}} \equiv\left(\frac{1}{1-\omega_{\mathrm{II}}}\right)_{r=c+0}
$$

and so on.

We can solve equation (45) for $C_{0}$ :

$$
C_{0}=-\frac{G}{\Delta}\left\{\frac{3 D(1-K C)[(1-G)(3 A-2 C)-(1-F) A]}{2(1-G)[3(1-K C)(A-C)-2 D C(1-3 K C)]+4(1-F) A D(1-3 K C)}+1\right\},
$$

where

$$
\Delta=\frac{\zeta^{2}}{\xi^{2}}, \quad \zeta=\Phi^{\Lambda} \text { and } \xi=\Theta^{\Gamma}
$$

with

$$
\begin{gathered}
\Phi=\frac{\gamma+\delta C}{\gamma+\delta A}, \quad \Lambda=-\frac{8 \pi H}{\delta}\left(\frac{\delta+3 K \gamma}{\gamma K+\delta}\right), \quad \Theta=\frac{1-K C}{1-K A}, \quad \Gamma=\frac{16 \pi H}{\gamma K+\delta} \\
D=g(1-2 M / C)^{1 / 2}-1
\end{gathered}
$$




$$
\begin{gathered}
G=(1-2 M / C), \\
H=\frac{A(1-F)-C(1-G)}{24 \pi(A-C)}, \quad K=\frac{4 \Omega-3}{3 A(4 \Omega-1)}, \quad F=\left(1-\frac{2 \bar{M}}{A}\right), \\
\left.m(0) \equiv \tilde{m}_{\mathrm{II}}\right|_{\substack{r=a \\
u=0}}, \quad M=\frac{\tilde{m}_{\mathrm{I}}[u, c(u)]}{m(0)}, \quad \bar{M}=\frac{\tilde{m}_{\mathrm{II}}[u, a(u)]}{m(0)} .
\end{gathered}
$$

We have changed $A$ to $a / m(0), C$ to $c / m(0)$, and $u$ to $u / m(0)$.

So far the set of unknown functions of $u$ reduce to

$$
A, F, \Omega, C, G, D, E, \Omega_{\mathrm{I}}, \Omega_{\mathrm{II}}, \epsilon_{\mathrm{I}}, \epsilon_{\mathrm{II}} .
$$

For them we have the three surface equations at $r=a(u)$ and the Rankine-Hugoniot conditions (45) (or [47]) and (46). Furthermore, we can obtain expressions for $\Omega_{\mathrm{I}}$ and $\Omega_{\mathrm{II}}$ from the field equations (3)-(5). Next, using the field equation (2) evaluated at each side of the shock, instead of the condition (46), we arrive at the equations (see Appendix):

$$
\dot{D}=\frac{T \pm \sqrt{T^{2}-4 \Sigma \Xi}}{2 \Sigma},
$$

and

$$
\dot{G}=\frac{-\phi \pm \sqrt{\phi^{2}-4 \chi \eta}}{2 \chi}
$$

Thus we are left with six equations for nine unknown functions of $u$.

In this particular example we assume the luminosity $E$ to be Gaussian so that the total radiated mass is a fraction (1/100 here) of the initial mass. Furthermore we also assume a pulse of radiation at $r=c+0$. Finally, $\epsilon_{\mathrm{I}}$ and $\epsilon_{\mathrm{II}}$ are related via a constant "opacity" parameter $\alpha$, as

$$
\epsilon_{\mathrm{I}}=\alpha \epsilon_{\mathrm{II}} \text {. }
$$

Additional comments on this particular choice of functions will be given in $\S \mathrm{V}$.

We are prepared to integrate the full system of surface equations and therefore to find the profile of matter variables for different pieces of material.

We have integrated the system for several sets of initial data. From the cases examined below the outcomes of the exploding sphere strongly depend upon the following parameters:

i) The mass of the inner core;

ii) The moment of occurrence of the peak of the pulse;

iii) The "opacity" of the shock.

More specifically, we have integrated for the following sets of initial data:

Set 1:

$$
\begin{aligned}
& \left.A\right|_{u=0}=20.0, \\
& \left.F\right|_{u=0}=0.9, \\
& \left.G\right|_{u=0}=0.9,
\end{aligned}
$$

Peak at $u=15.0$,

$$
\alpha=1.0
$$

Set 2:

$$
\begin{aligned}
& \left.A\right|_{u=0}=20.0, \\
& \left.F\right|_{u=0}=0.9, \\
& \left.G\right|_{u=0}=0.9,
\end{aligned}
$$

Peak at $u=15.0$,

$$
\begin{gathered}
\left.\Omega\right|_{u=0}=1.00, \\
\left.C\right|_{u=0}=12.0, \\
\left.D\right|_{u=0}=-0.598, \\
\sigma=1.2,
\end{gathered}
$$

$$
\begin{aligned}
& \left.\Omega\right|_{u=0}=1.00, \\
& \left.C\right|_{u=0}=12.0, \\
& \left.D\right|_{u=0}=-0.598,
\end{aligned}
$$$$
\sigma=1.2
$$

$\alpha=1.5$ 
Set 3:

$$
\begin{aligned}
& \left.A\right|_{u=0}=20.0, \\
& \left.F\right|_{u=0}=0.9, \\
& \left.G\right|_{u=0}=0.9,
\end{aligned}
$$

Peak at $u=15.0$,

$$
\alpha=0.5 \text {. }
$$

$$
\begin{aligned}
\left.\Omega\right|_{u=0} & =1.00, \\
\left.C\right|_{u=0} & =12.0, \\
\left.D\right|_{u=0} & =-0.598, \\
\sigma & =1.2,
\end{aligned}
$$

Set 4:

$$
\begin{array}{rlrl}
\left.A\right|_{u=0}=20.0, & \left.\Omega\right|_{u=0}=1.00, \\
\left.F\right|_{u=0}=0.9, & \left.C\right|_{u=0}=12.0, \\
\left.G\right|_{u=0}=0.9, & \left.D\right|_{u=0}=-0.598, \\
\text { Pak at } u=15.0, & \alpha=-1.0 . & \sigma=1.2,
\end{array}
$$

Set 5:

$$
\begin{aligned}
& \left.A\right|_{u=0}=20.0, \\
& \left.F\right|_{u=0}=0.9, \\
& \left.G\right|_{u=0}=0.924,
\end{aligned}
$$

Peak at $u=15.0$,

$$
\begin{aligned}
\left.\Omega\right|_{u=0} & =1.00, \\
\left.C\right|_{u=0} & =12.0, \\
\left.D\right|_{u=0} & =-0.598, \\
\sigma & =1.2,
\end{aligned}
$$

$$
\alpha=1.0 \text {. }
$$

Set 6:

$$
\begin{aligned}
& \left.A\right|_{u=0}=20.0, \\
& \left.F\right|_{u=0}=0.9, \\
& \left.G\right|_{u=0}=0.890,
\end{aligned}
$$

Peak at $u=15.0$,

$$
\begin{aligned}
\left.\Omega\right|_{u=0} & =1.00, \\
\left.C\right|_{u=0} & =12.0, \\
\left.D\right|_{u=0} & =-0.598, \\
\sigma & =1.2,
\end{aligned}
$$

$$
\alpha=1.0 \text {. }
$$

Set 7:
$\left.A\right|_{u=0}=20.0$,
$\left.\Omega\right|_{u=0}=1.00$,
$\left.F\right|_{u=0}=0.9$,
$\left.C\right|_{u=0}=12.0$,
$\left.G\right|_{u=0}=0.9$,
$\left.D\right|_{u=0}=-0.598$,
$\sigma=1.2$,
Peak at $u=35.0$,

$\alpha=1.0$. 
Set 8:

$$
\begin{aligned}
& \left.A\right|_{u=0}=20.0, \\
& \left.F\right|_{u=0}=0.9, \\
& \left.G\right|_{u=0}=0.9,
\end{aligned}
$$

Peak at $u=25.0$,

$$
\begin{aligned}
\left.\Omega\right|_{u=0} & =1.00, \\
\left.C\right|_{u=0} & =12.0, \\
\left.D\right|_{u=0} & =-0.598 \\
\sigma & =1.2,
\end{aligned}
$$

$$
\alpha=1.0 \text {. }
$$

In all these sets inner pulse carries $10^{-5}$ of the initial sphere mass, while the outer one ejects $10^{-2}$. Here we have used $\sigma$ as a measure of the width of the pulse of radiation, which comes from the usual variance, $\sigma^{2}$, of the Gaussian probability density function (see, e.g., Meyer 1975, p. 225).

The first set of data leads to an exploding sphere (see Fig. 2.) Observe that even though the shock recedes, the outer parts of the sphere bounce and expand after some time; we shall discuss this point later. We have monitored matter variables (Figs. 3-6) through four windows, $r / a=0.25,0.55,0.75$, and 0.95 . Besides, Figures $7 a-7 c$ show the evolution for some of them measured at both sides of the shock.

Sets $1,2,3$, and 4 make evident the influence of the "opacity" of the shock. It is worth mentioning that $\alpha$ not only comprises actual shock opacity $(\alpha>1.0)$, but also its radiance to either region $(\alpha<1.0 \rightarrow$ II, or $\alpha<0 \rightarrow$ I). Figure 8 records some of these situations.

Another important factor in the evolution of the sphere happens to be the mass of the inner core. This can be seen from the result of the integration for sets 5 and 6 . We would expect that a denser inner core gives rise to more violent explosions, but, at the same time, massive cores enhance gravitational attraction. Figure 9 shows how this later case is determinant in our examples.

Finally, the influence of the position of the peak of radiation on the outcome of the evolution is illustrated by sets 1,7 , and 8 , and Figure 10 record this effect.

A more detailed discussion of these results is deferred to the next section.

\section{DISCUSSION OF THE RESULTS}

Originally, the example of the preceding section was conceived with the sole purpose of illustrating the method presented in $\S$ III. We harbored the hope (and still do) that astrophysicists with much better information on the process of collapse than we have could feed the method with the appropriate equation of state for the effective variables and an adequate set of initial data. However, the results which we found are (we believe) interesting enough by themselves to deserve discussion.

First of all, it should be noticed that in this case the shock not only does not reach the boundary surface at $r=a$, but recedes by the time the outer part is expanding. The stronger the shock recedes, the more violent exploding models one obtains (see Fig. 8).

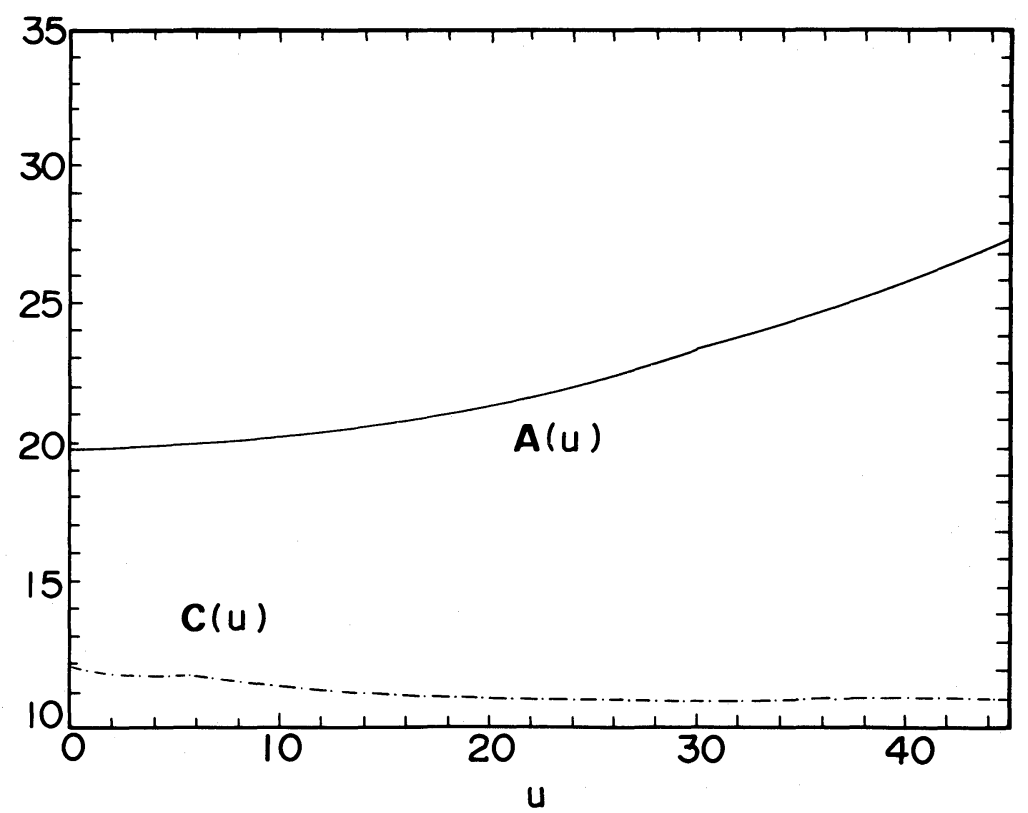

FIG. 2.-Time evolution of $A(u)$ and $C(u)$ for an exploding sphere. These are the evolutions of $A(u)$ and $C(u)$ for set 1 of initial data. The shock stalls and recedes, while $A(u)$ expands after some time. 


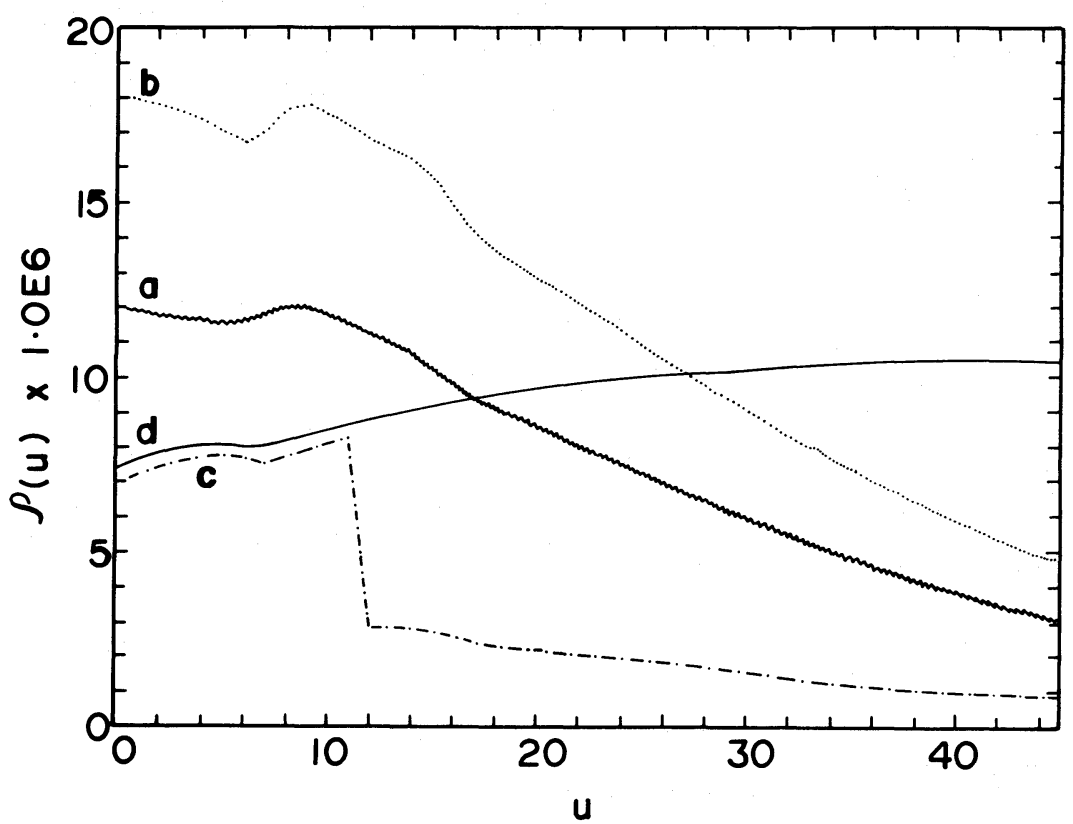

FIG. 3.-Evolution of density for set 1 of initial data, in four different shells. Values of the ratio $r / a$ for each curve are $a \rightarrow 0.95, b \rightarrow 0.75 . c \rightarrow 0.55$, and $d \rightarrow 0.25$. In order to obtain a better monitoring scale, we have multiplied values of curves $c$ and $d$ by $1.0 \times 10^{5}$, and $a$ and $b$ by $1.0 \times 10^{6}$. It is clear from curves 0.75 and 0.95 that the decrease of density at region II follows the emission of the radiation pulse from the inner core. Density curves for $r / a=0.25$ show that density of the inner region raises while the shock recedes.

These facts play central roles in a recently proposed supernova model (Bethe and Wilson 1985). Furthermore, our hydrodynamical picture is very similar with that of Bethe and Wilson. Indeed, observe from Figures 3-6 that prior to the bounce of a given shell, there is a decrease of density and a significant flux of energy across it. All this creates perfect conditions, as concluded by Bethe and Wilson, for the escaping of some part of the sphere from gravitational attraction. It is interesting to note that in our framework the bifurcation zone does not take place on the shock surface; instead, it occurs in some other part of the outer mantle. It seems to happen as if a "primary shock" [the one at $r=c(u)]$ induces, while contracting, an expanding bifurcation surface.

The importance of shock luminosity and neutrino luminosity at the shock front in the evolution of a supernova had been stressed by several authors (Bruenn, Buchler, and Yueh 1978; Van Riper 1982). They had also outlined some difficulties modeling these processes related to the complex and not well-known shock structure. We introduce an "opacity" parameter $\alpha$ which, relating the energy flux density at both sides of the shock, roughly incorporates into our scheme different possible behaviors of the radiation flux

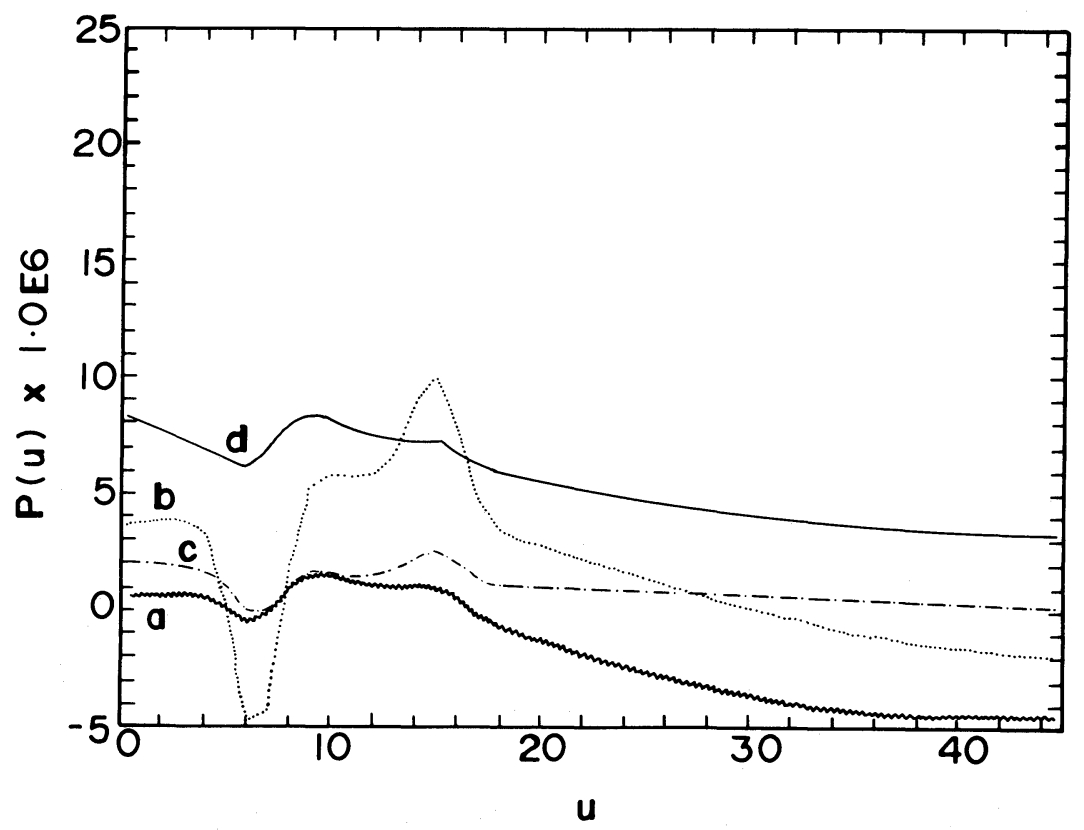

Fig. 4.-Evolution of pressure for set 1 of initial data, in four different shells. Values of the ratio $r / a$ for each curve are $a \rightarrow 0.95, b \rightarrow 0.75, c \rightarrow 0.55$, and $d \rightarrow 0.25$. 


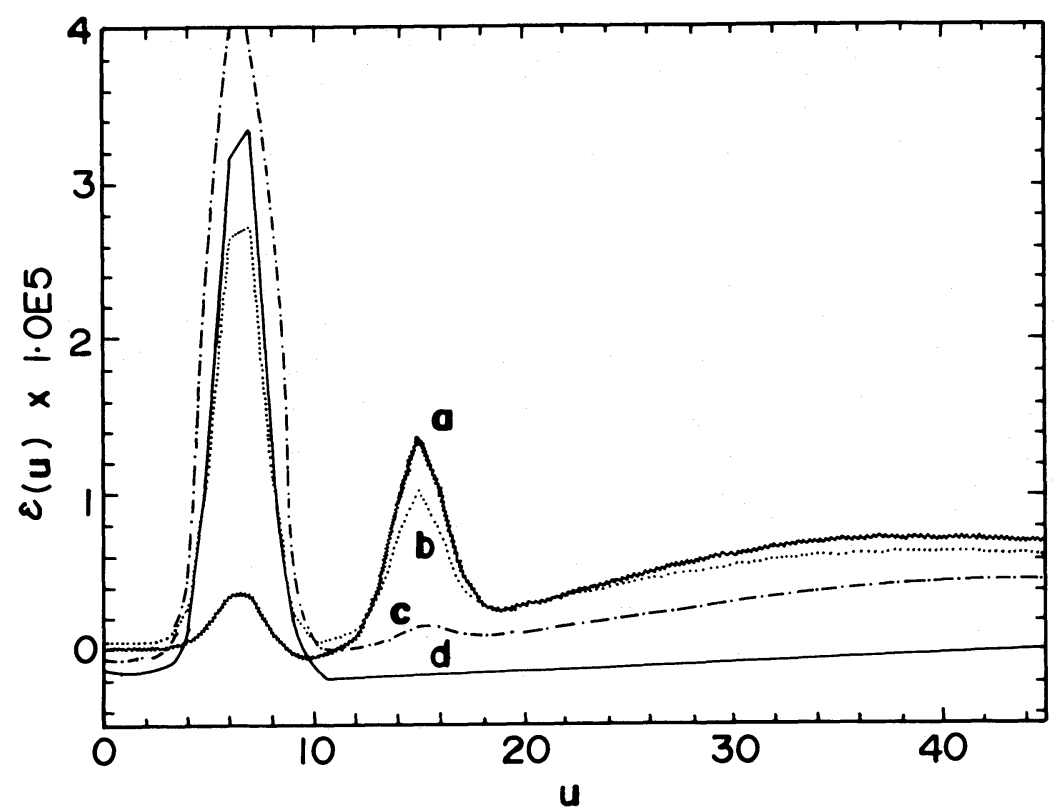

Fig. 5.-Evolution of energy flux for set 1 of initial data. Four different shells monitor this evolution. Here $r / a$ values are the same as in Figs. 3 and $4: a \rightarrow 0.95$, $b \rightarrow 0.75, c \rightarrow 0.55$, and $d \rightarrow 0.25$. Production and ejection of the pulse of radiation are clear from these curves. In spite of the pulse being ejected, outer shells still radiate.

across it. Shock opacity, transparency, and radiance to one or both regions were considered through $\alpha>1.0, \alpha=1.0, \alpha<1.0$, $\alpha<0.0$, respectively. This is a very crude approximation, with the sole virtue of simplicity. In spite of this, we obtain, as expected (Van Riper 1982; Kahana, Baron, and Cooperstein 1984), that a radiant shock induces fainter explosions, while opacity generates violent ones (see Fig. 8). Probably a function $\alpha=\alpha(u)$, instead of a constant value, would be more suitable describing supernova scenario. It remains to be seen which of these cases and what kind of opacity function illustrate better a physical situation.

Other parameters which critically influence the outcome of the evolution are the mass of the inner core (Fig. 9) and the moment of the occurrence of the peak of the pulse (Fig. 10). Massive cores enhance gravitational attraction, and this inhibits violent explosions. This picture closely resembles the one suggested by Cooperstein, Bethe, and Brown (1984); there the reduction in the core mass can lead to viable explosions.

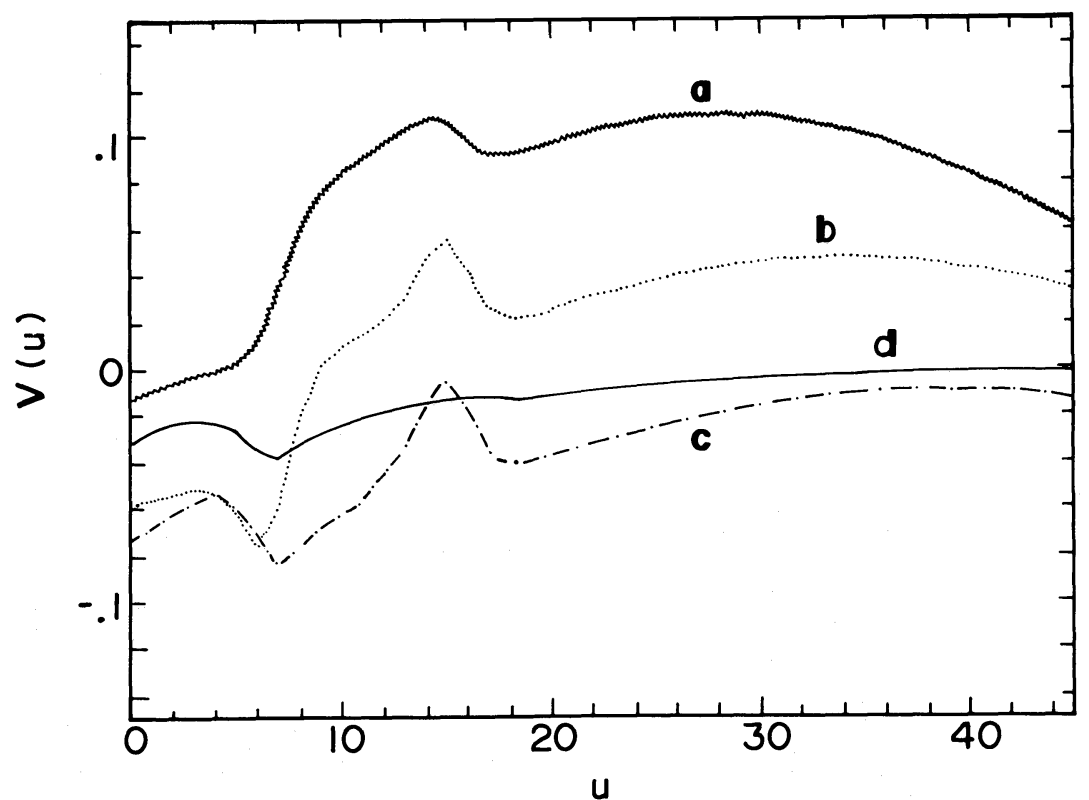

Fig. 6. - Evolution of mass velocity for set 1 of initial data. Four different mass layers are monitored. Values of $r / a$ ratio are $a \rightarrow 0.95, b \rightarrow 0.75, c \rightarrow 0.55$, and $d \rightarrow 0.25$. At the beginning of the evolution, curves for all shells display the collapse of these layers at both regions. After about five time units, the period in which the pulse begins to be emitted from inner core, the outer layers accelerate, and after the radiation pulse is ejected they expand continuously. 


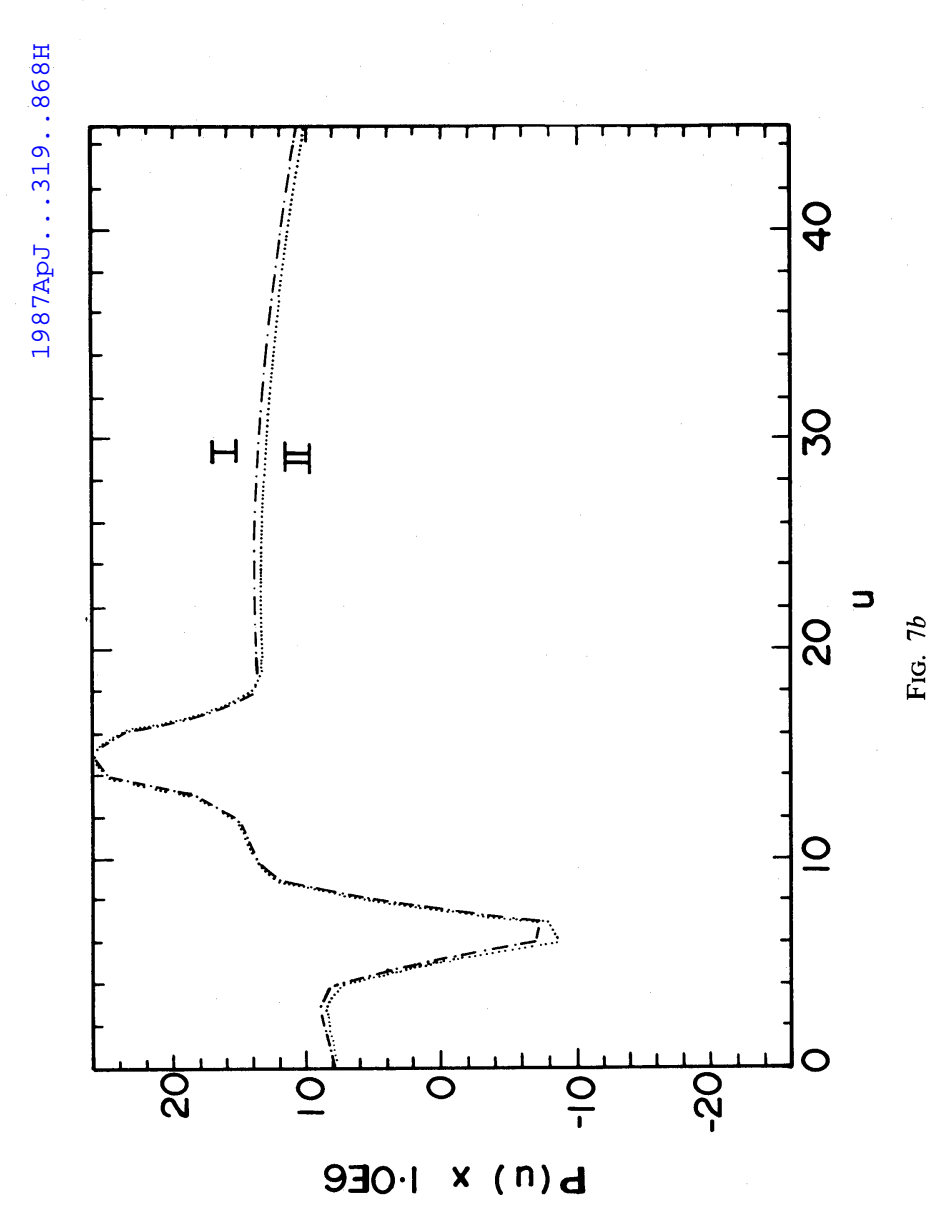

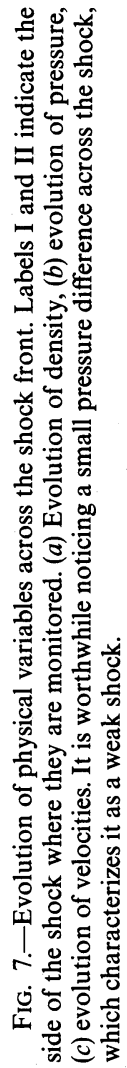
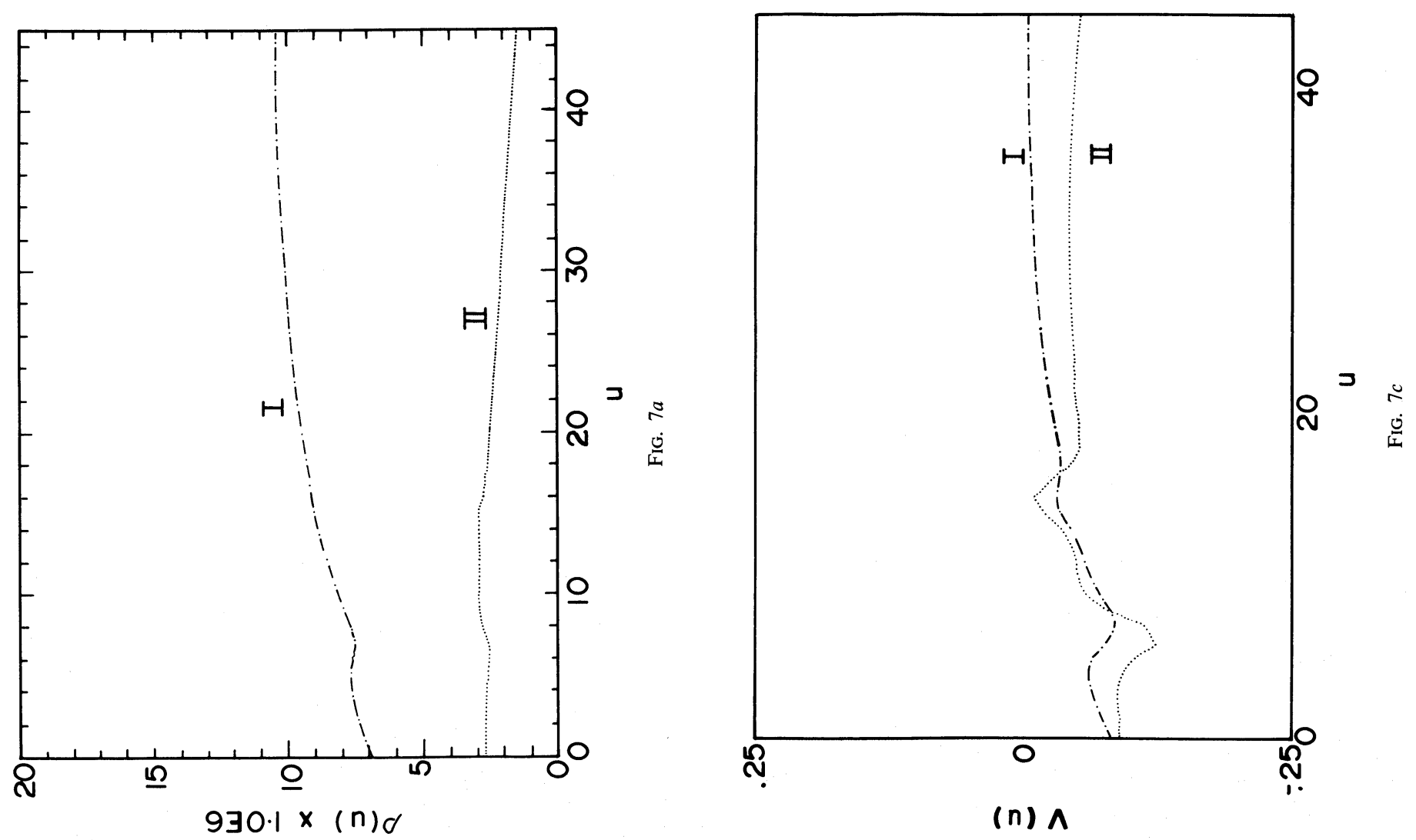

(C) American Astronomical Society - Provided by the NASA Astrophysics Data System 


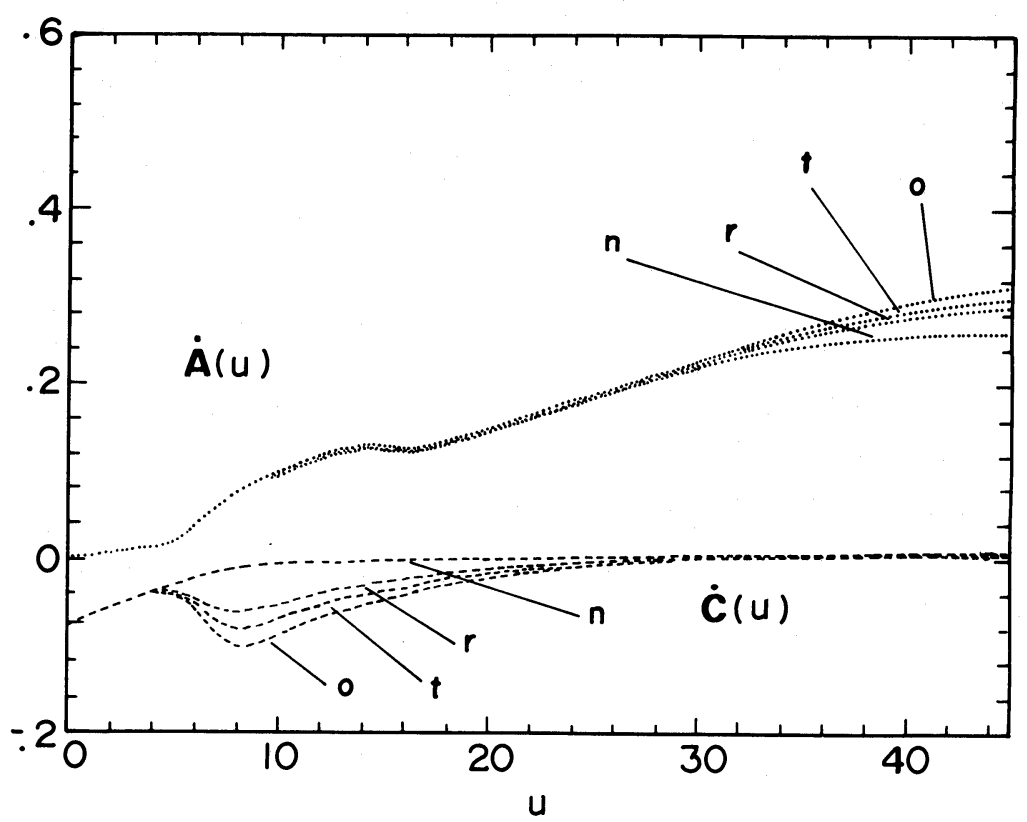

Fig. 8.-Influence of opacity. Curves are labeled as follows: $o \rightarrow \alpha=1.5, r \rightarrow \alpha=0.5, t \rightarrow \alpha=1.0$, and $n \rightarrow \alpha=-1.0$. They record the velocities of the front and the boundary surface. The $o$ curve illustrates how deeper recessions are associated with more violent expansions.

It is also worthwhile to notice, we believe, that it is a weak shock which emerges from our models, and this is clear from Figures $7 a-7 c$.

We would like to conclude this work with some general considerations on the method proposed. First, it should be clear to the reader that the "philosophy" of the procedure consists in avoiding a head-on integration of the Einstein equations. Instead, a heuristic relation among the matter variables is assumed. This Ansatz, which is suggested by the form of the field equations, links continuously our models with the corresponding static solutions. Besides the Ansatz on the effective variables, our solutions are restricted by the junction conditions at the boundary surface and shock front. As a consequence of all this, we are led to a system of ordinary differential equations for functions depending on the timelike coordinate. The integration is carried out numerically using the well-known RKF45 subroutine. This was developed by Watts and Shampine in 1974 (for details see, Forsythe, Malcolm and Moler 1977, p. 129), based on Fehlberg fourth-fifth order Runge-Kutta method (Fehlberg 1970). Second, we do not need to introduce an artificial viscosity to deal with shocks, avoiding at once all the reported inconveniences inherent to that method (Bruenn, Buchler, and Yueh 1978). Another point that deserves attention is the choice of the functions which should be given

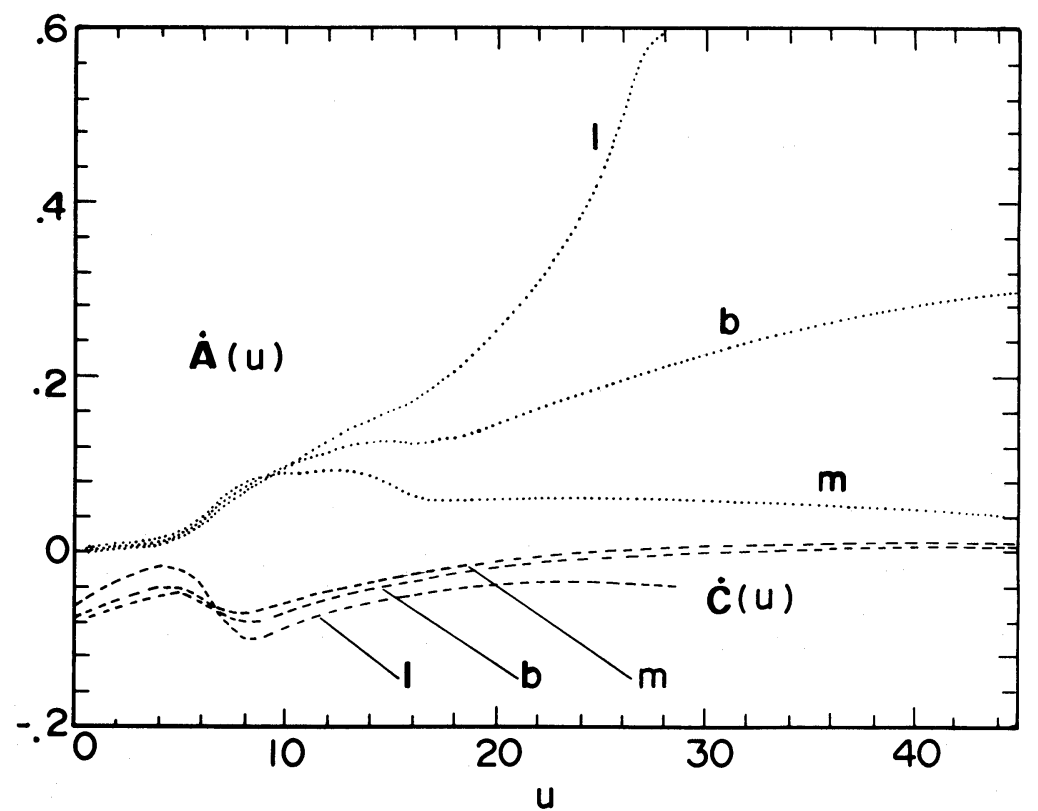

Fig. 9. - Influence of core mass. The labels of these curves represent $l \rightarrow G=0.924, b \rightarrow G=0.900$, and $m \rightarrow G=0.890$. It is clear that massive cores inhibit violent expansions because they enhance gravitational attraction. 


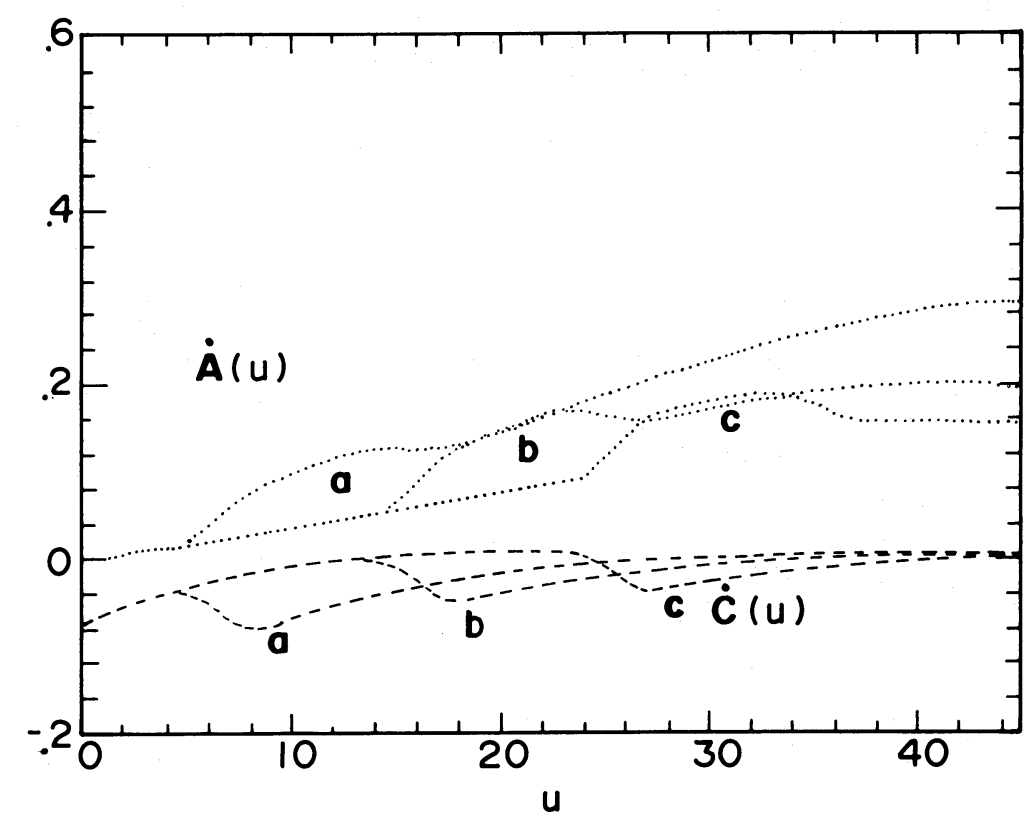

FIG. 10.-Influence of the peak pulse "position." Curves a, b, and c display the importance of the moment of occurrence for the peak of radiation. Notice that the earlier it is ejected the stronger the expansion is. This obviously connects radiation with expansion.

explicitly in order to integrate the surface equations. We believe that the most relevant observable in a supernova event should be the profile of the neutrino luminosity. This is the reason we give explicitly the function $E$. However, as far as we know, the neutrino pulse from a supernova has not been experimentally detected. Thus from the observational point of view it could be, just as well, justified to choose any other function of $u(A, \Omega, C$, etc.). Once again, we would like to hear the opinion on this point from specialists. Also, in connection with this question, observe that condition (52), which gives $\epsilon_{\mathrm{II}}$, also allows to introduce absorption and/or scattering effects on the neutrino flux.

We thank Gustavo Bruzual A. from Centro de Investigaciones de Astronomia CIDA for providing the graphical software, GRAFICO $^{\mathrm{TM}}$, used through this work. One of us (L. H.) gratefully acknowledges the financial support from Fundación Polar. This work has been supported in part by Consejo de Desarrollo Cientifico y Humanistico de la Universidad de Los Andes, Mérida, under project C 187-82.

\section{APPENDIX}

\section{EVALUATION OF $\Omega_{\text {I }}$ AND $\Omega_{\text {II }}$}

We sketch here some of the intermediate calculations coming up from the evaluation of $\Omega_{1}$ and $\Omega_{\mathrm{II}}$.

The field equation (2) for $r=c$ is

$$
\frac{\rho+P \omega^{2}}{1-\omega^{2}}+\epsilon=\frac{1}{4 \pi c^{2}[1-2 \tilde{m}(u, c) / c]}\left\{-\tilde{m}_{0 c} e^{-2 \beta(u, c)}+\left[1-\frac{2 \tilde{m}(u, c)}{c}\right] \tilde{m}_{1 c}\right\} .
$$

Expanding $\tilde{m}(u, r)$ around $r=c$, we have

$$
\tilde{m}(u, r)=\tilde{m}(u, c)+(r-c) \tilde{m}_{1 c}+\ldots,
$$

and

$$
\tilde{m}_{0 c}=m_{0}+\dot{c} \tilde{m}_{1 c} .
$$

The field equation (A1), written in the language of effective variables and using equation (3) for $r=c$, becomes

$$
(\tilde{\rho}+\tilde{P})(\Omega-1) \Omega+\tilde{\rho}+\epsilon=\Psi \tilde{\rho}-\frac{1}{4 \pi c^{2}[1-2 \tilde{m}(u, c) / c]} \tilde{m}_{0} e^{-2 \beta(u, c)} .
$$

We can also evaluate this last equation at either side of the shock; thus,

$$
\left(\Omega_{s}-1\right) \Omega_{s}=\left(\tilde{\rho}_{s}+\tilde{P}_{s}\right)^{-1}\left[(\Psi-1) \rho_{s}-\epsilon_{s}-\frac{\tilde{m}_{0} e^{-2 \beta_{s}}}{4 \pi c^{2}\left(1-2 \tilde{m}_{s} / c\right)}\right],
$$

with $s=$ I, II. 
We can express $\Omega_{1}$ and $\Omega_{\mathrm{II}}$, using equations (3), (4), and (5), as

$$
\Omega_{s}=\frac{\left(\tilde{m}_{s, 1} / 4 \pi c^{2}\right)-\left(\beta_{s, 01} / 4 \pi\right) e^{-2 \beta_{s}}+(1 / 8 \pi)\left(1-2 \tilde{m}_{s} / c\right)\left(2 \beta_{s, 11}+4\left(\beta_{s, 1}\right)^{2}-\beta_{s, 1} / c\right)}{\left(\beta_{s, 1} / 2 \pi c\right)\left(1-2 \tilde{m}_{s} / c\right)}+\frac{3\left(1-2 \tilde{m}_{s, 1}\right)-\tilde{m}_{s, 11}}{4\left(1-2 \tilde{m}_{s} / c\right)},
$$

and $s=$ I, II.

Note that here we have explicitly used a comma for denoting differentiation, in hopes to avoid any possible confusion with the subscripts.

Substituting in equation (A3) the expressions for $\beta_{s}, \beta_{s, 1}, \beta_{s, 11}, \beta_{s, 01}, \tilde{m}_{s}, \tilde{m}_{s, 1}$ and $\tilde{m}_{s, 11}$ in terms of the dimensionless variables, we can shape these equations, in the following form:

$$
\Omega_{\mathrm{I}}=Y \dot{D}+Z, \quad \Omega_{\mathrm{II}}=V \dot{G}+\mathrm{W} ;
$$

thus, we can also shape equation (A2) as

$$
(Y \dot{D}+Z)(Y \dot{D}+Z-1)=X \quad \text { for } s=\mathrm{I},
$$

and

$$
(V \dot{G}+W)(V \dot{G}+W-1)=L \dot{G}+U \quad \text { for } s=\mathrm{II} .
$$

The right sides of these equations are, expressed in terms of the selected variables, the right side of equation (A2) for the corresponding value of the subscript. The particular expressions for functions $V, W, L, U, Y, Z$, and $X$ will be given at the end of this appendix.

Finally, the first-order differential equation is written as a solution of a quadratic, namely

$$
\Sigma \dot{D}^{2}+T \dot{D}+\Xi=0 \leftrightarrow \dot{D}=\frac{-T \pm \sqrt{T^{2}-4 \Sigma \Xi}}{2 \Sigma},
$$

where

$$
\Sigma=Y^{2}, \quad T=Y(2 Z-1), \quad \Xi=Z(Z-1)-X,
$$

and

$$
\chi \dot{G}^{2}+\phi \dot{G}+\eta=0 \leftrightarrow \dot{G}=\frac{-\phi \pm \sqrt{\phi^{2}-4 \chi \eta}}{2 \chi}
$$

where

$$
\chi=V^{2}, \quad \phi=V(2 W-1)-L, \quad \eta=W(W-1)-U .
$$

\section{THE GENERALIZED TOLMAN-OPPENHEIMER-VOLKOFF EQUATION}

We present here the particular form of equation (19), the generalized Tolman-Oppenheimer-Volkoff equation, for $r=a(u)$. It can be written in the following form:

$$
\frac{\dot{\Omega}}{\Omega}+\tau_{1} \frac{\dot{F}}{F}+\tau_{2} \frac{\dot{C}}{C}+\tau_{3} \frac{\dot{G}}{G}+\tau_{4}=0,
$$

where

$$
\begin{aligned}
\tau_{1}=1+ & \frac{A F}{A(1-F)-C(1-G)}, \quad \tau_{2}=\frac{A C(F-G)}{(A-C)[A(1-F)-C(1-G)]}, \quad \tau_{3}=-\frac{C G}{A(1-F)-C(1-G)}, \\
\tau_{4}= & \frac{(\Omega-1)}{2 \Omega A}\left[24 \pi H(3 \Omega-1)-\Omega(F+3)-4 F \Omega^{2}\right]+\frac{1}{8 A}\left\{-3 F+4(1-F)\left[\Omega-\frac{24 \pi H}{(1-F)}(\Omega-1)\right]\right\} \\
& +\frac{F(\Omega-1)}{A}\left\{2+\frac{A C(F-G)}{(A-C)[A(1-F)-C(1-G)]}\right\} .
\end{aligned}
$$

III. EXPRESSIONS FOR $U, L, W, V, X, Y$, and $Z$

$U=\frac{\Delta(1-K C)\left[(G-F) A \dot{C}-2 G E_{\mathrm{II}}(A-C)\right]}{4 G(1-3 K C)[A(1-F)-C(1-G)]}, \quad L=\frac{\Delta(1-K C)(A-C) C}{4 G(1-3 K C)[A(1-F)-C(1-G)]}, \quad W=\tau_{1} \frac{\dot{F}}{F}-\tau_{2} \frac{\dot{C}}{C}+\tau_{3} \frac{\dot{A}}{A}+\tau_{4}$ 
where

$$
\begin{aligned}
\tau_{1}= & \frac{\Delta C F A}{A(1-F)-C(1-G)}-\frac{8 \Omega \Delta K C}{G(4 \Omega-1)(4 \Omega-3)(1-K C)(1-3 K C)}\left[1+\frac{A F}{A(1-F)-C(1-G)}\right], \\
\tau_{2}= & \frac{\Delta A(G-F) C^{2}}{2 G(A-C)[A(1-F)-C(1-G)]}+\frac{\Delta C[(1-G)(24 A-23 C)-A(1-F)]}{48(A-C) G^{2}} \\
& +\frac{8 \Omega \Delta K C}{G(4 \Omega-1)(4 \Omega-3)(1-K C)(1-3 K C)}\left\{\frac{A C(F-G)}{(A-C)[A(1-F)-C(1-G)]}\right\}, \\
\tau_{3}= & \frac{\Delta A(G-F) C^{2}}{2 G(A-C)[A(1-F)-C(1-G)]}-\frac{\Delta K C}{G(1-K C)(1-3 K C)} \\
& -\frac{8 \Omega \Delta K C}{G(4 \Omega-1)(4 \Omega-3)(1-K C)(1-3 K C)}\left\{2+\frac{A C(F-G)}{(A-C)[A(1-F)-C(1-G)]}\right\} \\
\tau_{4}= & \frac{3(A-C)[2 G+(1-K C)]+(1-9 K C)[A(1-F)-C(1-G)]}{12 G(1-K C)(A-C)}-\frac{8 \Omega \Delta C C}{G(4 \Omega-1)(4 \Omega-3)(1-K C)(1-3 K C)} \\
& \left.\times \llbracket \frac{(\Omega-1)}{2 \Omega A}\left[24 \pi H(3 \Omega-1)-\Omega(3+F)-4 F \Omega^{2}\right]-\frac{3 F}{8 A}\left\{1-\frac{4(1-F)}{3 F}\left[\Omega-\frac{24 \pi H(\Omega-1)}{(1-F)}\right]\right\}\right],
\end{aligned}
$$

with

$$
\begin{gathered}
H=\frac{A(1-F)-C(1-G)}{24 \pi(A-C)}, \quad K=\frac{4 \Omega-3}{3 A(4 \Omega-1)}, \\
V=\frac{\Delta C}{2 G^{2}}\left\{\frac{[A(1-F)-C](4 \Omega-1)(4 \Omega-3)(1-K C)(1-3 K C)+16 \Omega K C G}{(4 \Omega-1)(4 \Omega-3)(1-K C)(1-3 K C)[A(1-F)-C(1-G)]}\right\}, \\
X=D\left\{\frac{E_{\mathrm{I}}}{(1-G)}-\frac{C \Delta}{2 G}\left[\frac{2 \dot{C}}{C}+\frac{\dot{G}}{(1-G)}\right]\right\}, \quad Y=\frac{\Delta C}{2 D G}, \\
Z=1+\frac{\Delta C}{2 D G^{2}}\left\{\frac{D \dot{G}}{(1-G)}+\frac{\dot{C}}{C}[2 D+(D+1)(1-G)]\right\} .
\end{gathered}
$$

\section{REFERENCES}

Arnett, W. D. 1966, Canadian J. Phys., 44, 2553.

1979, in Sources of Gravitational Radiation, ed. L. Smarr (Cambridge:

Cambridge University Press), p. 311.

Bethe, H., and Wilson, J. R. 1985, Ap. J., 295, 14

Bondi, H. 1964, Proc. Roy. Soc. London A, 281, 39

Brown, G. E., Bethe, H. A., and Baym, G. 1982, Nucl. Phys. A., 375, 481

Bruenn, S. W., Arnett, W. D., and Schramm, D. N. 1977, Ap. J., 213, 213.

Bruenn, S. W., Buchler, F. R., and Yueh, W. R. 1978, Ap. J. (Letters), 221, L86.

Colgate, S. A., and Johnson, M. H. 1960, Phys. Rev. Letters, 5, 253.

Colgate, S. A., and White, R. H. 1966, Ap. J., 143, 626.

Cooperstein, J., Bethe, H. A., and Brown, G. E. 1984, Nucl. Phys. A, 429, 527.

Fehlberg, E. 1970, Computing, 6, 61.

Forsythe, G. E., Malcolm, M. A., and Moler, C. B. 1977, Computer Methods for Mathematical Computations (Englewood Cliffs: Prentice-Hall).

Herrera, L., and Jiménez, J. 1983, Phys. Rev. D, 28, 2987.

Herrera, L., Jiménez, J., and Ruggeri, G. 1980, Phys. Rev. D, 22, 2305.

Kahana, S., Baron, E., and Cooperstein, J. 1984, in Problems of Collapse and

Numerical Relativity, ed. M. Signore (Dordrecht: Reidel), p. 163.
Kazanas, D., and Schramm, D. N. 1979, in Sources of Gravitational Radiation, ed. L. Smarr (Cambridge: Cambridge University Press), p. 345.

Lichtenstadt, I., Sack, N., and Bludman, S. A. 1980, Ap. J., $237,903$.

Meyer, S. 1975, Data Analysis for Scientists and Engineers (New York: Wiley).

Migdal, A. B., Chernoutsan, A. I., and Mishustin, I. N. 1979, Phys. Lett. B, 83, 158.

Schramm, D. N., and Arnett, W. D. 1975, Ap. J., 198, 629.

Schwartz, R. A. 1967, Ann. Phys., 43, 42.

Taub, A. H. 1948, Phys. Rev., 74, 328.

. 1983, in Proc. 3d. Marcel Grossman Meeting on General Relativity, ed. H. Ning (Amsterdam: North Holland; Beijing: Science Press), p. 165.

Tolman, R. 1939, Phys. Rev., 55, 364.

Van Riper, K. A. 1979, Ap. J., 232, 558.

. 1980, Ap. J., 240, 658.

Wilson, J. R. 1971, Ap. J., 163, 209.

. 1979, in Sources of Gravitational Radiation, ed. L. Smarr (Cambridge: Cambridge University Press), p. 335.

LuIS HerRera: Apartado 80793, Caracas 1080A, Venezuela

LuIS NúÑEZ: Departamento de Fisica, Facultad de Ciencias, Universidad Central de Venezuela, Caracas-Venezuela 